\title{
Sec6 I $\beta$, a subunit of the Sec6 I protein translocation channel at the Endoplasmic Reticulum, is involved in the transport of Gurken to the plasma membrane.
} Anshuman Kelkar*1,2 and Bernhard Dobberstein ${ }^{1}$

Address: ${ }^{1 Z e n t r u m}$ für Molekulare Biologie Universitat Heidelberg, Im Neuenheimer Feld 282, Heidelberg 69120, Germany and 2The Rockefeller University and Howard Hughes Medical Institute, 1230 York Avenue, New York, NY 10065, USA

Email: Anshuman Kelkar* - akelkar@rockefeller.edu; Bernhard Dobberstein - dobberstein@zmbh.uni-heidelberg.de

* Corresponding author

Published: 18 February 2009

BMC Cell Biology 2009, 10:1I doi:10.1186/147I-212I-10-II
Received: 10 August 2008

Accepted: 18 February 2009

This article is available from: http://www.biomedcentral.com/I47I-2/2I//0/II

(c) 2009 Kelkar and Dobberstein; licensee BioMed Central Ltd.

This is an Open Access article distributed under the terms of the Creative Commons Attribution License (http://creativecommons.org/licenses/by/2.0), which permits unrestricted use, distribution, and reproduction in any medium, provided the original work is properly cited.

\begin{abstract}
Background: Protein translocation across the membrane of the Endoplasmic Reticulum (ER) is the first step in the biogenesis of secretory and membrane proteins. Proteins enter the ER by the Sec6I translocon, a proteinaceous channel composed of three subunits, $\alpha, \beta$ and $\gamma$. While it is known that Sec6l $\alpha$ forms the actual channel, the function of the other two subunits remains to be characterized.
\end{abstract}

Results: In the present study we have investigated the function of Sec6/ $\beta$ in Drosophila melanogaster. We describe its role in the plasma membrane traffic of Gurken, the ligand for the Epidermal Growth Factor (EGF) receptor in the oocyte. Germline clones of the mutant allele of Sec6I $\beta$ show normal translocation of Gurken into the ER and transport to the Golgi complex, but further traffic to the plasma membrane is impeded. The defect in plasma membrane traffic due to absence of Sec6I $\beta$ is specific for Gurken and is not due to a general trafficking defect.

Conclusion: Based on our study we conclude that Sec6I $\beta$, which is part of the ER protein translocation channel affects a post-ER step during Gurken trafficking to the plasma membrane. We propose an additional role of Sec6I $\beta$ beyond protein translocation into the ER.

\section{Background}

Translocation of proteins across the membrane of the Endoplasmic Reticulum (ER) is the first step in the biogenesis of secretory and membrane proteins. The nascent polypeptide chains of these proteins enter the ER via a proteinaceous channel called the Sec61p complex and gain access to the secretory pathway. If the mature protein does not possess specific signals for retention in the ER the protein exits the ER and is transported to the Golgi complex. The protein then moves along the Golgi complex till it reaches the trans-Golgi region. From the trans-Golgi region the protein is either transported to endocytic organelles or to the plasma membrane.

The Sec61 translocon is a heterotrimer constituted by $\alpha, \beta$ and $\gamma$ subunits. According to the crystal structure of the SecY complex from Methanococcus jannaschii, which is presumed to represent the fundamental structure of the Sec61 protein translocation channel at the ER, the $\alpha$ subunit, forms the actual channel. The $\beta$ and the $\gamma$ subunits are associated with the channel on the periphery and are in contact with the lipid bilayer [1]. Data obtained from 
in-vitro experiments using liposomes reconstituted with individual components of the Sec61p channel indicate that $\operatorname{Sec} 61 \alpha$ and $\gamma$ are necessary for the protein translocation per-se [2]. Sec61 $\beta$, on the other hand, is dispensable [2]. In accordance with the in-vitro data, in S. cerevisiae mutating $\alpha$ and $\gamma$ subunits of Sec61p is lethal whereas mutating Sec $61 \beta$ results only in a mild temperature sensitive phenotype $[3,4]$.

In contrast to the observations in S. cerevisiae, Sec61 $\beta$ is essential in Drosophila melanogaster; embryos homozygous for an allele with a P-element insertion before the transcription start site $\left(\sec 61 \beta^{\mathrm{p} 1}\right)$ die at the end of embryogenesis, at stage 17 [5]. The sec61 $\beta^{\text {P1 }}$ allele was used for generation of the maternal germline clones causing depletion of Sec $61 \beta$ protein from the ovaries. These flies produced embryos that died before stage 17 with the dorsal appendages completely or partially fused [5]. This dorsal appendage phenotype indicates perturbations in the dorsal-ventral axis with increased ventralization of the oocyte. Mutations that inhibit the EGF receptor signalling during oogenesis are known to cause this phenotype [6]. The only known EGF receptor ligand in the oocyte is Gurken. Gurken protein is synthesized in the oocyte but signals to the EGF receptor on the surrounding follicle cells [7]. Asymmetric signalling by Gurken to the follicle cells in the oocyte constitutes the first step towards formation of the dorsal-ventral axis in the embryo [7]. This asymmetric signalling is initiated when the Gurken mRNA is exclusively localized to the posterior end of the oocyte during early stages of oogenesis and to the anterior-dorsal end of the oocyte during the later stages [7]. Local translation of the mRNA results in synthesis of Gurken as a type I membrane protein in the ER. Like other members of the EGFR ligand family, transport of Gurken from the ER to the plasma membrane is highly regulated. An ER resident chaperone, Star, mediates the exit of Gurken from the ER [8]. During the transport through the secretory pathway to the plasma membrane Gurken protein is cleaved within the trans-membrane region by action of a member of the Rhomboid (Rho) family of intra-membrane proteases, called Brother of Rhomboid $[8,9]$. Cornichon ensures packaging of the Gurken protein in specialized COPII vesicles [10]. The soluble protein is secreted from the oocyte and interacts with the cognate receptor in the neighbouring follicle cells [8].

In this study, we investigated the possibility that the dorsal appendage fusion phenotype observed in the embryos from the sec $61 \beta^{\mathrm{P} 1}$ germline clones is due to changes in the biosynthesis of Gurken. We analysed the localization of Gurken mRNA and Gurken protein in wild type and sec61 $\beta$ mutant egg chambers. We found that during different stages of oogenesis depletion of Sec61 $\beta$ from the oocytes results in reduced levels of Gurken at the plasma membrane and in the surrounding follicle cells. We also observed that reduced amounts of Sec $61 \beta$ protein does not affect the ER translocation of Gurken, majority of the Gurken protein can enter the ER and is successfully transported till the Golgi complex. Transport of Gurken protein beyond the Golgi complex is affected by lack of Sec61 $\beta$. This defect seems to be specific for Gurken since transport of a control protein is not affected. We propose that Sec61 $\beta$ plays an essential role during later stages of Gurken transport to the plasma membrane.

\section{Results \\ Expression of Sec6 I $\beta$ in wild type and sec6 I $\beta^{\mathrm{PI}}$ germline clones}

The $\sec 61 \beta^{\text {P1 }}$ allele has been reported to be a probable loss-of-function allele [5]. In order to characterise the allele in greater detail we generated an antibody against the N-terminus of Sec61 $\beta$. This antibody was used for western blot analysis using protein extracts from wild type ovaries and ovaries derived from female germline homozygous for $\sec 61 \beta^{P 1}$ (Fig. 1A). The amount of Sec61 $\beta$ protein is significantly reduced in ovaries derived from homozygous mutant clones as compared to the wild type ovaries. The small amount of Sec61 $\beta$ protein observed in sec $61 \beta^{P 1}$ homozygous clones most likely represents Sec $61 \beta$ in follicle cells. Follicle cells do not originate from the germ cell lineage and should retain normal amounts of $\operatorname{Sec} 61 \beta[11]$.

\section{Localization of the Gurken mRNA is unaffected in the germline clones of Sec6 I $\beta$}

The germline clones of the sec $61 \beta^{\mathrm{p} 1}$ allele generated embryos with fused dorsal appendages indicating perturbations in the dorsal-ventral axis [5]. The observed phenotype is strongly reminiscent of the dorsal-ventral axis defect found in the Gurken mutations. Asymmetric localization of the Gurken mRNA at the anterior-dorsal end of the ooycte is the first step towards establishment of the dorsal-ventral axis [12]. It has been proposed that the Gurken mRNA is closely associated with the ER and an ER protein could anchor the mRNA at the anterior-dorsal end of the oocyte [13].

We therefore examined the localization of Gurken mRNA in the stage 10 egg chambers by in situ hybridisation. In wild type oocytes Gurken mRNA localizes to the anteriordorsal end of the oocyte in close proximity to the oocyte nucleus as indicated in the cartoon (Fig. $1 \mathrm{~B}$ and $1 \mathrm{C}$ ). In sec61 $\beta$ mutant oocytes Gurken mRNA similarly localizes to the anterior-dorsal end of the oocyte (Fig 1C and 1D). Thus, the sec $61 \beta^{\text {P1 }}$ germline clones show no apparent difference in the localization of Gurken mRNA in oocytes. 

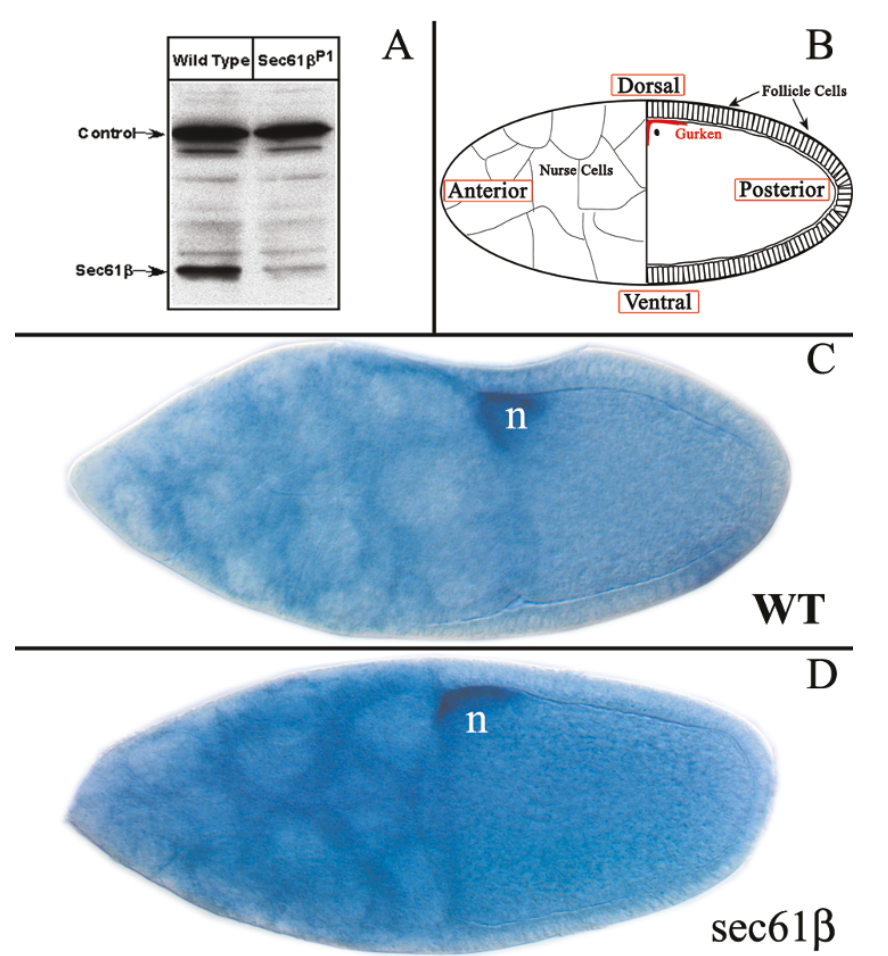

Figure I

Sec6 I $\beta$ protein and Gurken mRNA in ovaries from wild type and sec6I $\beta^{\text {PI }}$ germline clones. A) Ovaries were dissected from flies and lysed in buffer containing $2 \%$ SDS. Western blotting was performed using Sec6I $\beta$ antibody. A cross-reactive band serves as a loading control. B) Schematic representation of a stage 9-10 egg chamber with the oocyte abutting the 15 nurse cells and surrounded by a layer of somatic follicle cells. The dorsal/anterior corner is depicted in dark red. C-D) Drosophila egg chambers were processed for gurken RNA in-situ hybridization in wild type oocytes, C) and in oocytes mutant for Sec6I $\beta$, D). n represents the oocyte nucleus.

Germline clones of sec6 I $\beta^{\mathrm{PI}}$ allele show reduced levels of Gurken protein at the plasma membrane of the oocyte

Since the mRNA localization of Gurken was not affected by lack of Sec61 $\beta$, we investigated whether lack of Sec61 $\beta$ affects the translation of Gurken mRNA or events in the transport of Gurken protein to the plasma membrane.

We performed immuno-fluorescence analysis on wild type egg chambers or germline clones of $\sec 61 \beta^{\mathrm{p} 1}$ using a monoclonal antibody generated using the N-terminal extra-cellular domain of the Gurken protein (Developmental Studies Hybridoma Bank, [14]). The egg chambers were also stained with phalloidin, which binds actin localized below the plasma membrane and serves to delineate the plasma membrane. In stage 10 wild type oocytes Gurken protein localizes at the anterior-dorsal part of the oocyte in close proximity to the oocyte nucleus in precisely the same region as the mRNA (Fig. 2A). The mutant stage 10 oocytes also stains positive for Gurken, with the protein localized in the anterior-dorsal end of the oocyte (Fig. 2B). Upon analysis of the anterior-dorsal part of the oocytes at higher magnification we observed Gurken at two different locations in the oocyte, in punctuate structures in the cytoplasm between the plasma membrane and the nucleus and at the plasma membrane at the anterior-dorsal end of the oocyte, co-localizing with actin, directly opposite the follicle cells or the nurse cells (Fig. 2C). In the oocytes derived from the germline clones of $\sec 61 \beta^{P 1}$ the cytoplasmic pool of Gurken protein was observed at the anterior-dorsal part of the oocyte in punctuate structures similar to the wild type oocytes but the Gurken protein amount at the plasma membrane was drastically reduced (Fig. 2D). Reduction in Gurken amount occurs in the part of the plasma membrane that is in direct apposition to the follicle cells and the nurse cells (Fig. 2D). To rule out the possibility that the observed changes in Gurken localization observed were due to differences in the focal plane in view, we analysed series of optical sections from different depths of the wild type

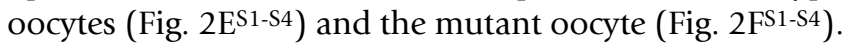
In all cases Gurken protein is excluded specifically from the plasma membrane.

We also observed staining for Gurken protein in distinct speckles inside the follicle cells at the anterior-dorsal end of the oocytes (Fig. 2G). These speckles most likely represent the protein that has been internalized by the follicle cells. In $\sec 61 \beta^{\mathrm{P} 1}$ germline clones on the other hand, Gurken staining in the follicle cells is rarely observed (Fig. $2 \mathrm{H})$. Taken together, these results demonstrate that egg chambers from the sec $61 \beta^{\mathrm{P} 1}$ germline clones have reduced levels of Gurken protein at the plasma membrane of the oocyte and in the surrounding follicle cells.

Gurken protein is also mis-localized during early oogenesis Gurken protein signals to the EGF receptor on the follicle cells at two different stages of oogenesis. During late oogenesis (stage 10-11) Gurken signals to follicle cells at the anterior-dorsal end of the oocyte. During earlier stages of oogenesis (stages 6-9) the oocyte nucleus and the Gurken mRNA are localized at the posterior part of the oocyte with Gurken protein signalling to the posterior follicle cells [12]. In order to investigate the localization of Gurken during early oogenesis we co-stained egg chambers during stage 6-9 of oogenesis with the anti-Gurken antibody and with phalloidin. The Gurken protein in the wild type egg chambers is localized in punctuate cytoplasmic structures towards the posterior part of the oocyte (Fig $3 \mathrm{~A}$ and $3 \mathrm{~B}$ ). We also observe Gurken staining in the posterior follicle cells. In the oocytes derived from sec $61 \beta^{\mathrm{p} 1}$ germline clones we observe Gurken in the oocyte cytoplasm similar to wild type oocytes (Fig 3C and 3D) but 

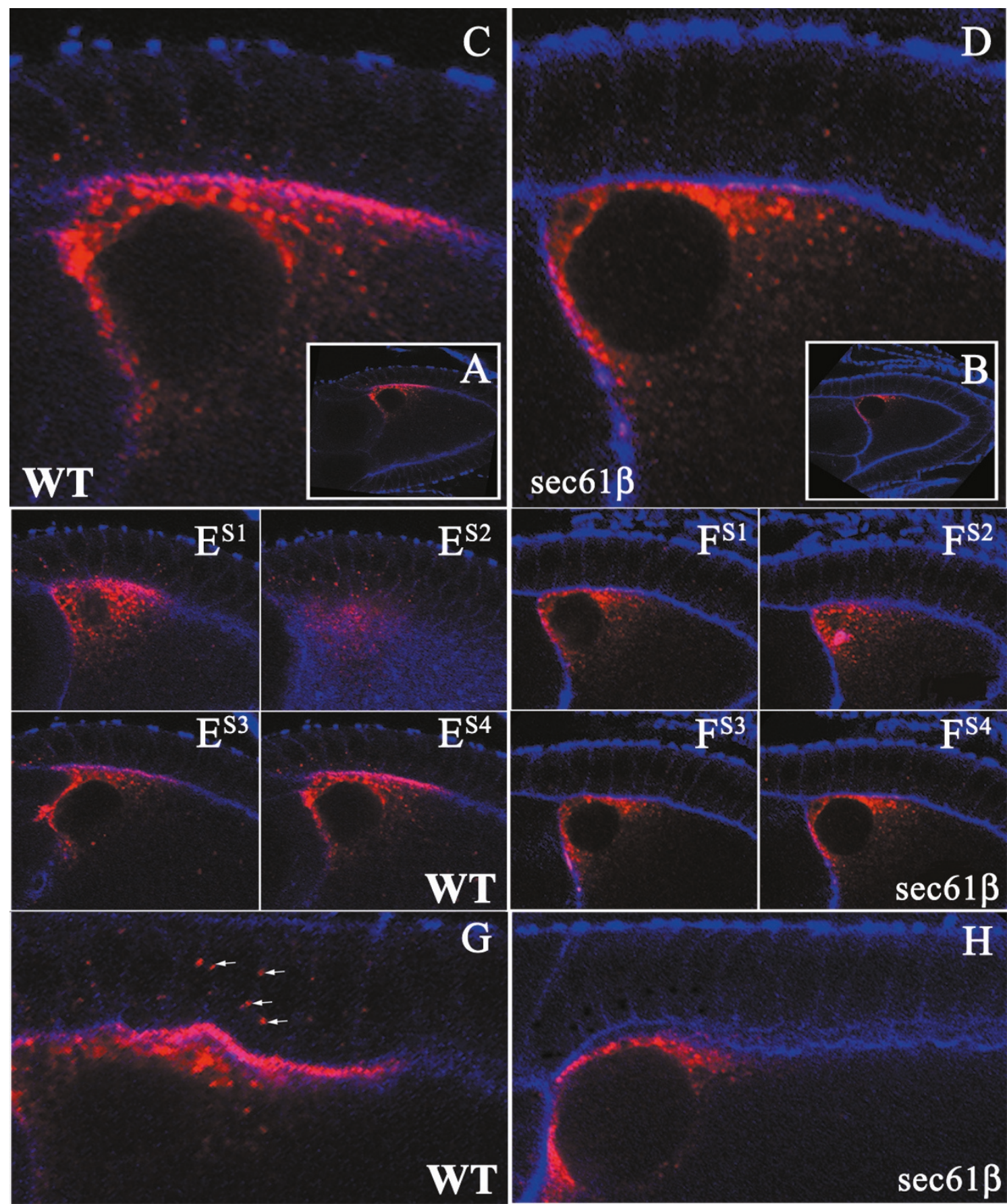

\section{Figure 2}

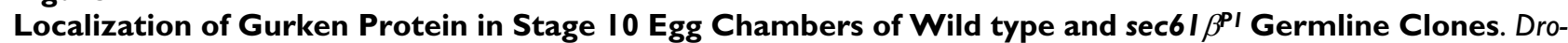
sophila egg chambers of the indicated genotype, wild type, (WT) or egg chambers mutant for sec6| $\beta$, (sec6| $\beta$ ), stained for actin using labelled Phalloidin (blue) and Gurken (red). A-B) Low magnification images of wild type (A) and sec6I $\beta$ mutant egg chamber (B) at stage 10; Gurken appears at the anterior-dorsal position. C-D) Magnification of the anterior-dorsal region of wild type and sec6I $\beta$ mutant oocytes. Gurken co-localizes with actin in the wild type oocytes (C), where as the sec6I $\beta$ mutant oocytes do not show this co-localization (D). $\mathrm{E}^{\mathrm{SI}-\mathrm{S4}}$-FSI-S4) Optical sections of the egg chambers as shown in $C$ and $D$, correspond to either wild type egg chambers ( $\mathrm{E}^{\mathrm{SI}-\mathrm{S} 4}$ ) or sec6I $\beta$ mutant egg chambers (FSI-S4). G-H) High magnification images of oocytes showing the region of the follicle cells from wild type $(G)$ and sec6I $\beta$ mutant egg chambers $(H)$. Gurken is seen in dot like structures within the follicle cells in the wild type egg chambers (indicated by arrows), these structures are absent in the mutant egg chambers. 


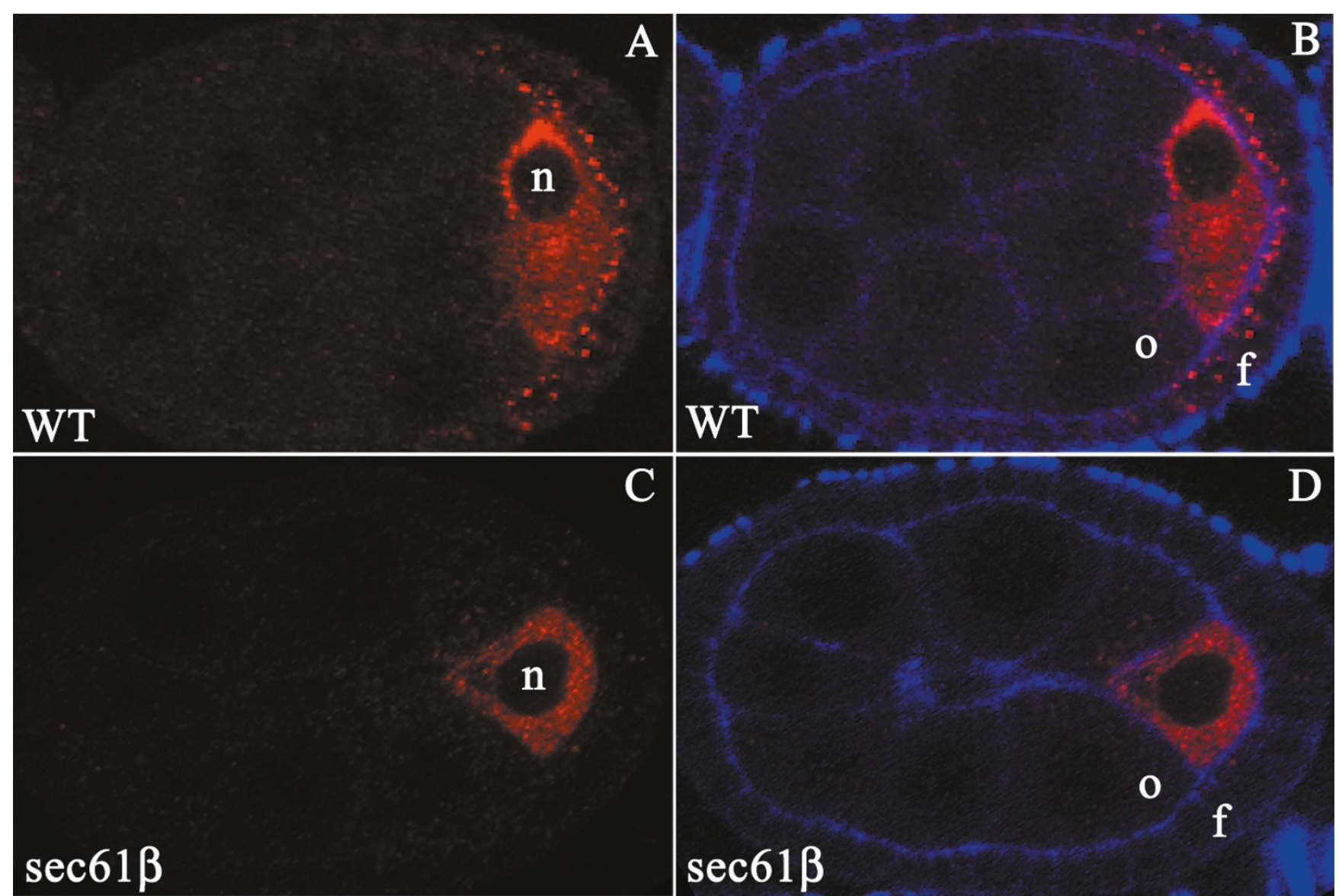

Figure 3

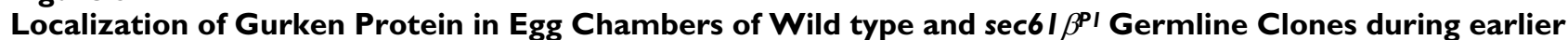
stages of Oogenesis. Drosophila egg chambers of the indicated genotype, wild type, (WT) or egg chambers mutant for $\sec 6 \mid \beta$, (sec6I $\beta)$, stained for actin using labelled Phalloidin (blue) and Gurken (red). A -B) Staining for Gurken protein (A) in the stage 6 oocyte from wild type, co-staining with actin (B) to delineate the follicle cells. C-D) Staining for Gurken protein (C) in sec6I $\beta$ mutant oocytes shows protein distributed all over the oocyte, co-staining with actin to delineate the follicle cells. $f$, indicates the follicle cells, o, the oocyte and $\mathrm{n}$, the oocyte nucleus.

not in the posterior follicle cells (Fig 3D). Thus, the Gurken trafficking defect is also observed during early stages of oogenesis.

\section{The general structure and function of ER remains unaffectedin sec6 I $\beta$ mutant oocytes}

Gurken is a type I membrane protein and the presence of the signal sequence suggests that the protein is most likely co-translationally translocated into the ER and transported along the secretory pathway to reach the plasma membrane. To investigate if the mislocalization of Gurken was due to a general impairment in structure and function of ER, we co-stained wild type and sec61 $\beta$ mutant egg chambers with the Gurken antibody and an antibody raised against the Boca protein that has been previously characterised as an ER resident protein in oocytes [15]. In wild type egg chambers during stage 9-10 of oogenesis we observe a diffused staining for Boca in a region below the plasma membrane throughout the oocyte (Figure 4A). Oocytes derived from the sec $61 \beta^{\mathrm{p} 1}$ germline clones show a very similar staining, suggesting that the overall organization of the ER remains largely unaffected by lack of Sec61 $\beta$ (Fig. 4C). Localization of Gurken in the wild type and the sec $61 \beta$ mutant oocytes is as observed previously (Fig 4B,D, and Fig 2).

The Sec61 translocon translocates a variety of secretory and membrane proteins into the ER. The reduction in the Gurken amounts at the plasma membrane in the germline clones of $\sec 61 \beta^{\mathrm{P} 1}$ could be due to a general defect in the 


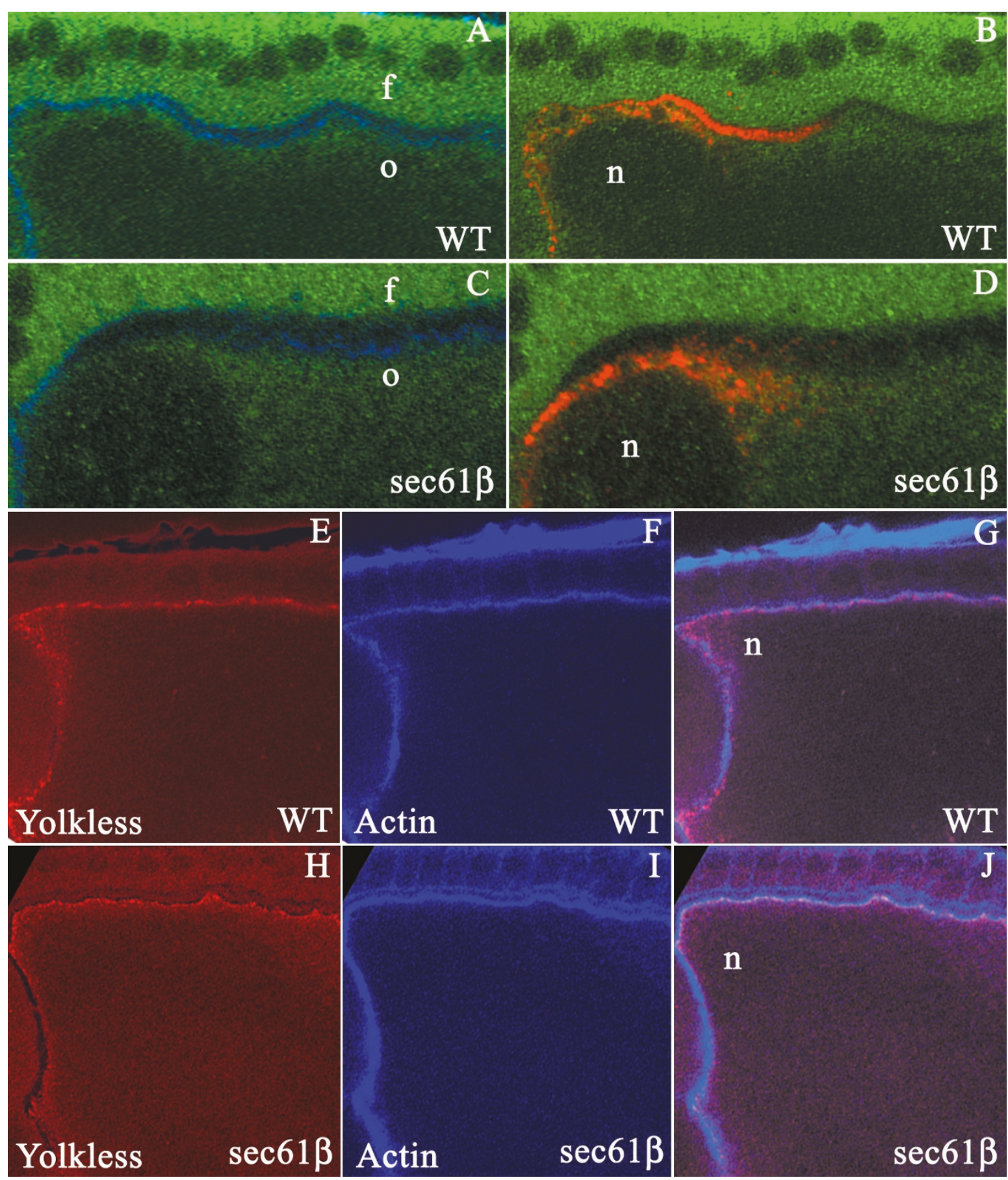

\section{Figure 4}

Analysis of ER structure and function in sec6 I $\beta$ mutant oocytes. A-D, Drosophila egg chambers of the indicated genotype, wild type, (WT) or egg chambers mutant for sec6I $\beta$, (sec6I $\beta$ ), stained for actin using labelled Phalloidin (blue), Gurken (red) and Boca (green). A and C) In oocytes from stage 10 egg chambers Boca stains a diffuse region below the plasma membrane that marks the ER in both the wild type $(A)$ and the sec6I $\beta^{P I}$ germline clones $(C)$. B and D) Gurken staining is observed either along the plasma membrane or in distinct punctate structures (B) or Gurken is excluded from the plasma membrane and is present exclusively in the cytoplam (D). $f$, indicates the follicle cells, o, the oocyte and $n$, the oocyte nucleus. E-J, Drosophila egg chambers of the indicated genotype, wild type, (WT) or egg chambers mutant for sec6I $\beta$, (sec6I $\beta$ ), stained for actin using labelled Phalloidin (blue) and Yolkless (red). In wild type egg chambers (E) or egg chambers mutant for sec6I $\beta(\mathrm{H}) \mathrm{YI}$ is at a peripheral location in the oocyte. Co-staining with phalloidin ( $\mathrm{F}$ and $\mathrm{I}$ ) shows that both in wild type oocytes and oocytes from the germline clones $\mathrm{YI}$ co-localizes at the plasma membrane ( $\mathrm{G}$ and $\mathrm{J})$. $\mathrm{n}$ indicates the position of the nucleus. 
plasma membrane trafficking. In order to investigate this possibility we examined the localization of another plasma membrane protein of the oocyte, Yolkless (Yl). Yl is a type I membrane protein expressed by the oocytes for the uptake of vitellogenins and yolk proteins during oogenesis [16]. We co-stained wild type and mutant oocytes for Yolkless (Yl) and actin. Upon staining we observed that $\mathrm{Yl}$ is localized to the oocyte plasma membrane in both the wild type egg chambers (Fig.4E-G) and $\sec 61 \beta^{\text {P1 }}$ homozygous clones (Fig.4H-J) during stage 9-10 of oogenesis, thus suggesting that Sec61 $\beta$ does not cause a general defect in protein trafficking.

\section{Sec6 I $\beta$ is not required for ER translocation}

Data obtained from immunofluorescence analysis in oocytes so far suggest that Sec61 $\beta$ was involved in the transport of the Gurken protein to the plasma membrane, however it was not clear which step of the transport process is affected. We wanted to further characterise this defect and to test the actual process of ER translocation using HeLa cells. Previous studies have also utilized the mammalian cell cultures system for investigating trafficking of EGFR ligands. Gurken and other EGF ligands such as Spitz and Keren can be expressed and secreted in mammalian cells. The process requires Star for ER exit and Rho for proteolytic processing, thus in part recapitulating the trafficking of EGFR ligands as it occurs in the fly system $[17,18]$. We used this system to examine if changes in the amount of Sec61 $\beta$ could affect the translocation of Gurken into the ER.

Gurken was transfected into HeLa cells, either alone or cotransfected with the regulatory proteins, Star and Rho. On western blot analysis of the whole cell lysate, Gurken is seen to migrate as a $45-47 \mathrm{KDa}$ protein (Fig. 5A). Upon co-transfection with Myc-tagged Star the position of the band is unchanged. When HA-tagged Rho is transfected, Gurken shows faster migration, which most likely represents the intra-membrane cleaved form of Gurken. Gurken is glycosylated as inferred from its sensitivity to Endo-glycosidase H, EndoH (Fig. 5A, right panel). Sensitivity to EndoH is retained even when Star and Rho are transfected along with Gurken indicating retention in the ER. Based on this data we think in this cell type Star does not cause an extensive Gurken export from the ER and Rho is able to process the Gurken within the ER. Expression of Star and Rhomboid was confirmed by western blot analysis using the anti-Myc and anti-HA antibody respectively (data not shown).

In order to investigate Gurken translocation into the ER in the absence of Sec61 $\beta$, a plasmid was transfected into HeLa cells that was designed to generate double stranded RNA oligo against Sec61 $\beta$ mRNA. As a control, a plasmid designed to generate a scrambled double stranded RNA oligo was also transfected. Three days after the initial transfection the cells were re-transfected with the Sec61 $\beta$ siRNA plasmid or the control siRNA plasmid, together with the expression plasmids for Gurken, Star and Rho. One day after the second round of transfections (and four days after the initial round of transfection) Sec $61 \beta$ protein is no longer detected by western blotting in cells transfected with the Sec $61 \beta$ siRNA plasmid, whereas cells transfected with the control plasmid retain normal level of Sec61 $\beta$ protein (Fig. 5B). Expression of Star and Rho is confirmed by western blot analysis using the anti-Myc and anti-HA antibodies respectively (Fig 5C).

ER translocation of Gurken was investigated as follows: a pulse analysis using ${ }^{35} \mathrm{~S}$-methionine was performed and the cells were harvested for immuno-precipitation and western blotting. The actual process of translocation and availability of Gurken for post-translocational processing was determined by the extent of glycosylated protein. Correctly translocated Gurken would be glycosylated in the $\mathrm{ER}$, on the other hand, conditions affecting translocation or downstream events would result in a species of Gurken that is unavailable for glycosylation.

The cell lysates after ${ }^{35} \mathrm{~S}$-methionine pulse were probed with antibody against the Gurken protein (Fig 5D). Gurken is visible in the processed, faster migrating form in control cell lysates and lysates with depeleted Sec61 $\beta$ protein. Gurken is EndoH sensitive in cells with normal and reduced Sec61 $\beta$ protein, hence glycosylated and present in the ER (Fig 5D). Guken protein was immuno-precipitated from control and Sec61 $\beta$ depleted cells; half of the immuno-precipitated sample was treated with EndoH and the other half was left untreated, both the samples were applied to SDS-PAGE. We observe that in lanes representing control and Sec61 $\beta$ depleted cells without EndoH treatment, the majority of Gurken protein runs as a single band along with minor lower molecular weight bands. (Fig. 5E, left half). Majority of the Gurken protein is also glycosylated since after EndoH treatment it runs at lower molecular weight (Fig. 5E, right half). It is important to note that the relative ratios of the glycosylated and unglycosylated forms of Gurken protein $(1 \mathrm{~g}$ and $0 \mathrm{~g}$ respectively) does not change in the absence of Sec61 $\beta$. Thus, the translocation of Gurken into the ER and posttranslocational processes such as glycoslation are not affected by the reduced level of Sec61 $1 \beta$. These experiments also show that cleavage of Gurken is unaffected by reduced amounts of Sec61 $\beta$, suggesting that Rho is translocated and functional (Fig 5D).

Taking together the data from co-staining in the oocytes and experiments in HeLa cells it seems that Gurken translocation into the ER and its post-translational modifica- 


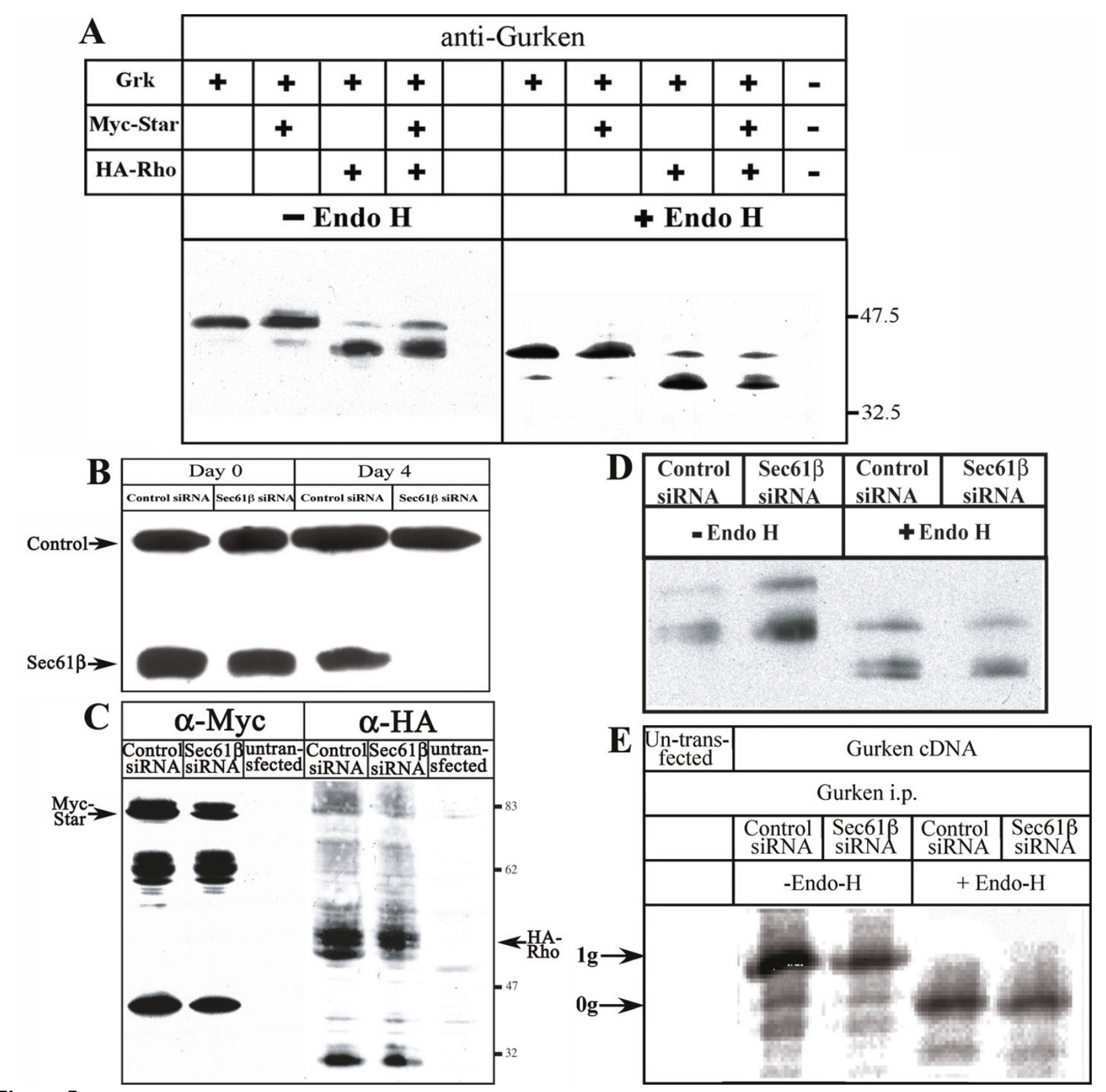

Figure 5

Expression of Gurken in HeLa cells. A) Plasmids encoding Gurken protein alone or in combination with Myc tagged Star and/or HA tagged Rho were transfected into HeLa cells as indicated on top of the figure or left untransfected. Aliquots of the cell lysates were applied to SDS-PAGE either directly or after being treated with EndoH. Western bloting was done using an antibody against Gurken. The numbers on the right indicate the molecular mass in kilo Daltons. B-E) HeLa cells were transfected with plasmid generating a double stranded RNA against Sec6 I $\beta$ or a control plasmid together with Gurken, Myc-Star and HA-Rho encoding plasmids as indicated. Cells lysates were applied to SDS-PAGE and western blot and probed with an antibody against Sec6 I $\beta(B)$. The lysates were also probed with antibodies against Myc and HA to detect expression of Star and Rho respectively (C). The same sets of cells growing on plates were used for a 15 min pulse with ${ }^{35} \mathbf{S}$ methionine and cell lysates were prepared. These lysates were used for western blots with or without EndoH treatment and probed with the Gurken antibody (D). The lysates after the pulse analysis were subjected to immuno-precipitation using anti-Gurken antibody and immuno-precipitated samples were applied to SDSPAGE either directly or after being treated with EndoH (E). Single glycosylated (0 g) and nonglycosylated (I g) forms of Gurken are indicated. 
tions remain unaffected by lack of Sec $61 \beta$. Sec $61 \beta$ seems to affect a step beyond the process of ER translocation.

\section{Gurken is transported till the Golgi complex in absence of Sec6I $\beta$}

Data obtained from immunofluorescence analysis in oocytes and in HeLa cells suggested that Sec61 $\beta$ was not involved in the transport of the Gurken protein into the $\mathrm{ER}$, on the contrary it appeared that it is required at a later, post-ER step of secretion. The finding that lack of a component of the translocation channel could affect a step beyond the ER translocation was indeed surprising. In order to investigate this further and we wanted to analyse the sub-cellular localization of Gurken in greater detail. In both wild type and mutant oocytes the cytoplasmic pool of Gurken protein is present in the peri-nuclear region and is organised into punctate structures. These Gurken containing punctate structures are interspaced within the dispersed ER in both wild type and sec $61 \beta$ mutant oocytes. In order to establish the identity of these punctuate structures we used antibody against the Lava Lamp (Lva) protein, a cis-Golgi marker [19] together with the Gurken antibody for immunofluorescence analysis. We observed that the punctate structures containing the Gurken protein frequently also contained the Lva protein and this was visible in either the wild type (Fig. 6A-C) or the $\sec 61 \beta^{\text {P1 }}$ oocytes (Fig. 6D-F).

We also analysed the sub-cellular localization of the Gurken protein using a ubiquitously expressing an EYFP tagged Golgi marker [20]. In both wild type (Fig 7A-C) and sec61 $\beta$ mutant oocytes (Fig 7D-F) we observed that a significant number of the punctate Gurken structures in the cytoplasm co-localized with the Golgi marker.

This suggests that in the sec $61 \beta$ mutants Gurken protein is able to enter the secretory pathway and traffic till cisGolgi, further transport to the plasma membrane however is impeded.

\section{Sec5 retains plasma membrane localization in sec6 I $\beta^{\mathrm{PI}}$ germline clones}

Based on the data obtained so far it seemed that Sec61 $\beta$ is involved in a post-translocation step during trafficking of a subset of proteins to the plasma membrane. We therefore wanted to analyse the possible post-translocational steps in Gurken transport that could be affected by lack of Sec61 $\beta$. Recent data suggests that Sec61 $\beta$ interacts with the components of the exocyst machinery in S. cerevisiae and in mammalian cells $[21,22]$. The exocyst complex is a multi-protein vesicle-tethering complex localized at the plasma membrane that plays a role in polarised protein transport [23]. Directional transport of Gurken to the plasma membrane requires the exocyst complex. Germline clones of a hypomorphic allele of a subunit of the exocyst complex, sec5 $5^{\mathrm{E} 13}$, shows lack of Gurken at the plasma membrane and cytoplasmic accumulation of Gurken [24]. Based on the interaction data and defects in Gurken localization in germline clones of $\sec 5^{\mathrm{E} 13}$ and $\sec 61 \beta^{\text {p1 }}$, we considered the possibility that failure of Gurken transport from the cytoplasmic location to the plasma membrane in Sec61 $\beta$ mutant egg chambers is due to lack of a functional exocyst.

In order to investigate this possibility we examined the localization of Sec5 protein in germline clones of $\sec 61 \beta^{\text {p1 }}$. We used an antibody against Sec5 to stain the egg chambers during stage 10 of oogenesis. In wild type oocytes Sec5 is localized to the plasma membrane (Fig. $8 \mathrm{~A}-\mathrm{C}$ ). In the oocytes from sec $61 \beta^{\text {P1 }}$ germline clones (Fig. $8 \mathrm{D}-\mathrm{E})$, the localization of Sec5 appears identical to the wild type oocyte. Thus, in absence of Sec61 $\beta$, Sec5 retains its plasma membrane localization.

\section{Discussion}

Sec6 I $\beta$ 's enigmatic presence in the translocation channel The mechanism of translocation of proteins across the ER membrane through the Sec 61 channel and the function of Sec61 $\beta$ in the translocation process has previously been investigated using reconstituted liposomes, isolated rough microsomes and $S$. cerevisiae system $[2,25,26]$. These studies suggest that for the mechanistic process of protein translocation Sec $61 \beta$ is dispensable. These data are also consistent with the crystal structure of the Sec61 complex, the $\alpha$ subunit of the complex forms the actual protein conducting channel whereas the $\beta$ and the $\gamma$ subunits are associated on the periphery [1]. Hence the question we wanted to address was: if Sec61 $\beta$ is not involved in the process of ER translocation how could we explain its essential role in Drosophila.

Major shortcomings in the previous studies investigating the function of Sec $61 \beta$ have been use of limited set of substrates for monitoring protein translocation and use of invitro models. In the present study we investigated in-vivo the molecular function of Sec61 $\beta$. We report that Sec61 $\beta$ is essential for the plasma membrane transport of the TGF $\alpha$-like growth factor, Gurken, in Drosophila oocytes. Reduced Gurken signalling in these oocytes results in embryonic lethality with severe defects in anterio-posterior axis determination. The surprising observation made in our study was that the defect in Gurken transport to the plasma membrane occurs not during ER translocation but during a later step in the transport process.

\section{Sec6 I $\beta$ affects post-ER transport of Gurken to the plasma membrane}

Having observed that the Gurken transport to the plasma membrane is affected in sec61 mutant oocytes we wanted to determine the step in the transport pathway 

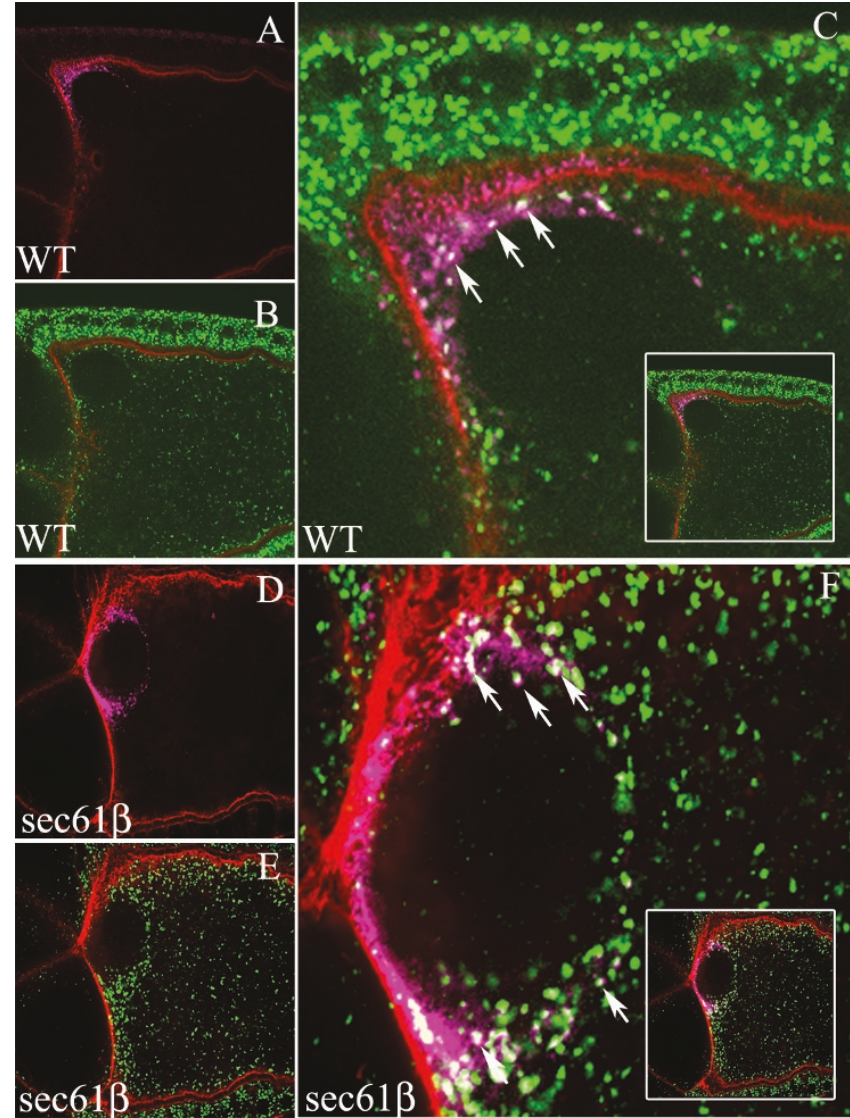

\section{Figure 6}

Co-localization of Gurken with Lva. Wild type egg chambers and egg chambers derived from germline clones of sec6 I $\beta^{\text {PI }}$ were stained with Gurken (Magenta), Lva (green) and Phalloindin (red). A-C) Wild type egg chambers with Gurken localized to the anterior-dorsal region of the ooctyte and also along the plasma membrane $(A)$ and Lva localized in the cytoplasm of the oocyte (B). Co-staining of Gurken shows frequent overlap of the signal for Gurken and Lva, as indicated by the arrows (C). D-F) Egg chambers derived from the germline clones of sec6 I $\beta^{P I}$ with Gurken localized to the anterior-dorsal end of the oocyte (D) and Lva localized in the cytoplasm of the oocyte (E). Co-staining of Gurken shows frequent overlap of the signal for Gurken and Lva, as indicated by the arrows $(F)$. Insets show whole of the co-stained oocyte.

that is affected by the absence of Sec61 3 . In the first set of experiments we investigated the possible role of Sec $61 \beta$ in ER translocation of Gurken using mammalian cells in culture. The mammalian cell system has been previously characterised to recapitulate the trafficking of EGFR ligands like Gurken and Spitz $[17,18]$. Gurken is a transmembrane protein with a signal sequence and would require the Sec 61 channel for the ER translocation. The ER translocation of Gurken, as determined by comparing the

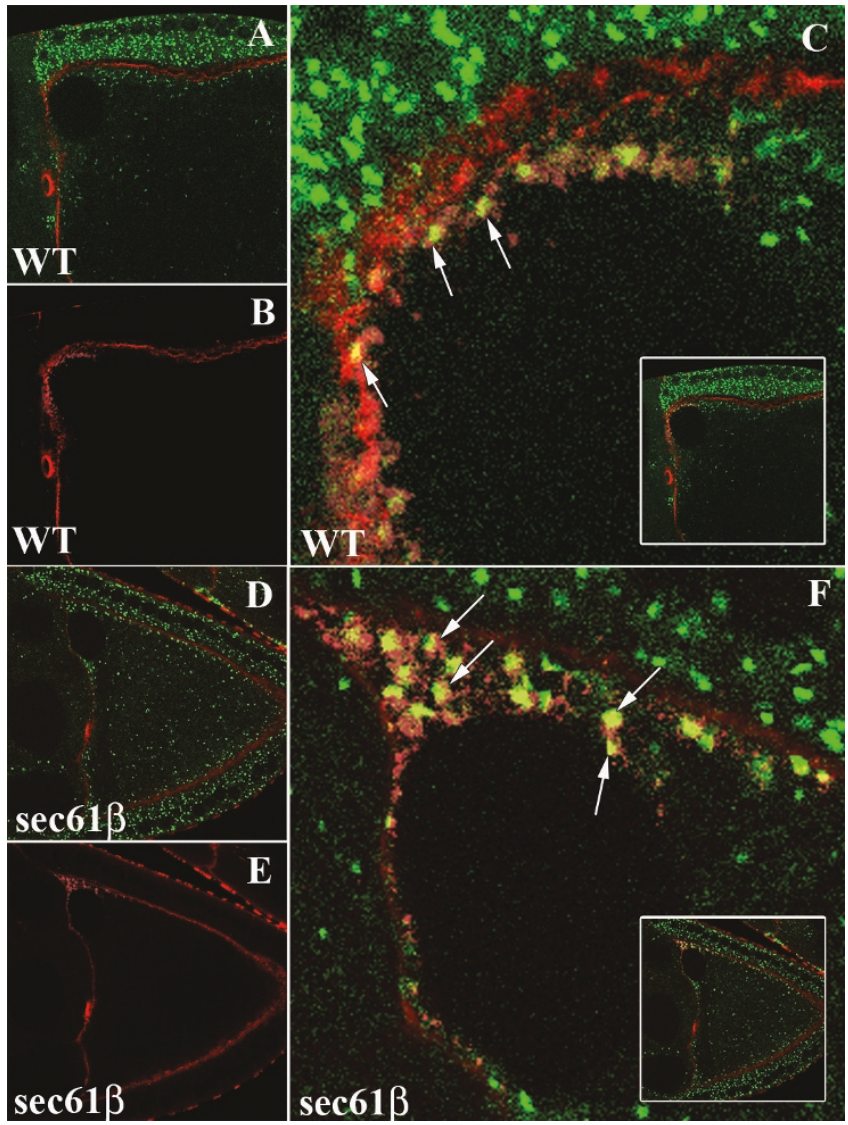

\section{Figure 7}

Co-localization of Gurken with a Golgi Marker. Wild type egg chambers and egg chambers derived from germline clones of sec6 I $\beta^{\mathrm{PI}}$ were stained with Gurken (Magenta) and Phalloidin (red). The ubiquitously expressed Golgi complex marker is in green. A-C) Wild type egg chambers expressing the Golgi marker $(A)$ and stained with Gurken, which is localized to the anterior-dorsal region of the oocyte $(B)$. A magnification of the anterior-dorsal region of the oocyte showing frequent co-localization of punctuate Gurken containing structures with the Golgi marker (C). The inset is same as $C$ but shows the whole egg chamber. D-F) Egg chambers mutant for sec6I $\beta$ expressing the Golgi marker (D) and stained with Gurken, which is localized to the anterior-dorsal region of the oocyte $(\mathrm{E})$. A magnification of the anterior-dorsal region of the oocyte showing frequent co-localization of punctuate Gurken containing structures with the Golgi marker $(F)$. The inset is same as $F$ but shows the whole egg chamber. Co-localization is indicated by white arrows.

relative amounts of glycosylated and un-glycosylated ${ }^{35} \mathrm{~S}$ Methionine labelled protein, was unaffected by the absence of Sec61 $\beta$. We also observed that post-translocational events co-ordinated with translocation, such as glycosylation, remain unperturbed by the absence of Sec $61 \beta$. Hence we confirm the previous observations that Sec61 $\beta$ indeed does not affect the ER translocation of Gurken. We 


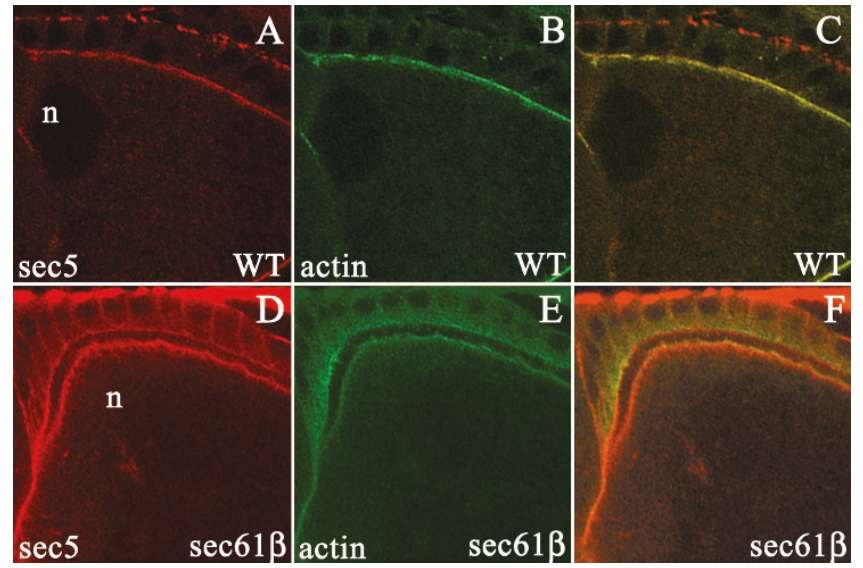

Figure 8

Localization of Sec5 in stage 10 oocytes. A-F) Wild type egg chambers or egg chambers from sec6 I $\beta^{p l}$ germline clones were stained with Sec5 (red) and Phalloidin (green). In wild type egg chambers $(A)$ or from sec6 I $\beta^{\text {PI }}$ germline clones (D) Sec5 is at a peripheral location in the oocyte. Co-staining with phalloidin ( $B$ and $E$ ) shows that both in wild type oocytes and oocytes from the germline clones Sec5 co-localizes at the plasma membrane. $\mathrm{n}$ indicates the position of the nucleus ( $\mathrm{C}$ and $\mathrm{F})$.

also observed that the proteolytic processing of Gurken by Rho occurred even in the absence of Sec $61 \beta$, suggesting an ER translocated and functional Rho.

Immuno-fluorescence data from oocytes revealed the cytosolic fraction of the Gurken protein in both wild type and sec $61 \beta$ mutant oocytes was intermingled with ER, as concluded from the co-staining with Boca, whereas the punctate Gurken structures contain the Golgi marker Lva. Similar results were obtained when Gurken was co-localized with Golgi marker, $p\{s q h$-EYFP-Golgi $\}$. Thus in the sec61 $\beta$ mutant oocytes Gurken is able to traffic till the Golgi complex, however further transport to the plasma membrane is impeded. The cytoplasmic punctuate organization of Gurken has been reported previously and shown to also contain dCOG, a component of the complex that tethers ER-derived vesicles to the Golgi membrane [27]. It is important to note that presence of the signal sequence suggests that Gurken most likely is cotranslationally translocated into the ER [2]. If a delay in translocation due to lack of $\operatorname{Sec} 61 \beta$ was to occur this would result in concomitant delay in translation and would result in highly reduced amounts of the Gurken protein in the oocytes. In our studies the amount of protein in the wild type and mutant oocyte does not appear to be drastically different, thus providing additional evidence for normal ER translocation of Gurken in the absence of Sec61 $\beta$.
Based on our data we think that Gurken transport to the plasma membrane occurs in two distinct steps. The first step is the translation of the localized Gurken mRNA followed by translocation into the ER and transport into the secretory pathway. Gurken is transported along the secretory pathway until it reaches the Golgi complex. This part of the Gurken transport process occurs independent of Sec61 $\beta$. The second phase in the Gurken secretion is transport of Gurken from Golgi to the plasma membrane, which our results indicate, requires Sec61 $\beta$.

As mentioned previously, there are two rounds of Gurken signalling during oogenesis. The first round of Gurken signalling occurs during the stage 7 of oogenesis, where Gurken signalling to the posterior follicle cells causes them to take the posterior fate. The follicle cells reciprocate the Gurken signalling from the oocyte by sending an as yet unknown signal back to the oocyte resulting in repolarization of the microtubule skeleton within the oocyte. This event is necessary for the migration of the nucleus to the anterior portion of the oocyte towards the later stages (stage 9) of oogenesis [28]. Although we observed the defect in the Gurken trafficking during the early stages of oogenesis we did not investigate the fate of the posterior follicle cells in the sec $61 \beta^{\text {p1 }}$ mutant oocytes. However, the migration of the nucleus to the anterior end of the oocyte was not affected, leading us to conclude that defect in Gurken trafficking at this stage does not result in a significant phenotype.

\section{Role of Sec6 I $\beta$ beyond ER translocation during Gurken Transport}

A hint at the post-ER function of Sec61 $\beta$ comes from two independent studies in mammalian cells in culture and in yeast. These studies report that Sec61 $\beta$ could physically and genetically interact with the members of the exocyst complex $[21,22]$. Exocyst is a complex of eight proteins that associate with the plasma membrane and localise to the regions of membrane addition [23]. In addition it has also been reported that Gurken transport to the plasma membrane requires the exocyst complex [24]. Germline clones of a hypomorphic allele of Sec5 ( $\left.\sec 5^{\text {E13 }}\right)$, a subunit of the exocyst complex, results in accumulation of Gurken in the cytoplasm [24]. Thus, it is possible that Gurken transport requires the Sec61 $\beta$-exocyst interaction and in absence of either of the two components Gurken is no longer transported to the plasma membrane. Various studies have indeed identified components of the exocyst at the ER, the interaction being cell and substrate dependent $[21,29]$. We did not observe changes in the plasma membrane localization of Sec5 or presence of Sec 5 at the ER in sec $61 \beta$ mutant oocytes. We believe that this is presumably due to the transient nature of the interactions and due to the fact that the exocyst contains sub-complexes that actually disassemble from the plasma mem- 
brane and Sec5 may not be part of this complex in the oocytes [30].

\section{Sec6 I $\beta$ required for a specific transport step}

The critical role of Gurken in development has mandated several regulatory steps in biosynthesis. The highly polarized Gurken transport process begins by localization of the Gurken mRNA at the anterior-dorsal end of the oocyte; this step is necessary but not sufficient to achieve polar transport [28]. Local translation of Gurken at the anterior-dorsal end of the oocyte translocates it into a ER that spans the entire oocyte. Mechanisms exit in the secretory pathway to prevent diffusion of the Gurken protein during its transport from the extended ER to the plasma membrane; these include selection of defined ER exit sites, specific interactions with specific cargo receptors and interactions with the exocyst $[24,27,31]$.

Transport along the secretory pathway involves export of Gurken protein from the ER a process mediated by Cornichon [10]. In our study we observe that even in the absense of Sec61 $\beta$ Gurken could be exported to the Golgi. This indicates that Cornichon is functional in the oocytes. This data also suggests that Star, another ER export factor, is also functional in these ovaries [18,32]. It is important to note this since both these proteins are transmembrane proteins and the fact that they are able to mediate Gurken export out of the ER and hence are functional which indicates that Sec61 $\beta$ does not affect the ER translocation of Star or Cornichon. The next step in the Gurken secretion process is its cleavage by the protease Rho $[17,18]$. While we could not analyse the cleavage state of Gurken in sec61 $\beta$ mutant ovaries, data from experiments in cultured cells show that even in the absence of Sec61 $\beta$ Rho is indeed able to cleave Gurken. In this system we also observe that the majority of the Gurken protein retains the ER specific glycosylation indicating continued retention in the ER. It is, however, still a substrate for Rho dependent cleavage. Processing of Gurken in the ER has previously been observed [31].

In mediating the traffic of different membrane proteins Sec61 $\beta$ seems to have a specific role in Gurken transport. The specificity of the role is illustrated by the observation that Yolkless does not require Sec61 $\beta$ for plasma membrane transport [24]. The role of Sec61 $\beta$ can be compared to that of Cornichon for Gurken transport and Boca for traffic of the LDL family of proteins. Both these protein like Sec61 $\beta$ are needed for the correct transport of individual proteins and appear to be present at an earlier stage of secretion. Gurken is retained in the oocyte in cornichon mutants although vitellogenesis proceeds normally [10]. Boca on the other hand is required for the trafficking of Yolkless and other LDL receptor family proteins but does not influence Gurken traffic [15]. All these factors are highly specific for certain proteins and do not seem to affect the general transport machinery. Although the role of Sec61 $\beta$ in Gurken transport seem to be indeed specific, we cannot completely rule out the possibility of an indirect effect where Sec61 $\beta$ affects translocation of a factor that is required in Golgi to plasma membrane transport of Gurken.

In development of an organism inter-cellular signalling occurs primarily by the interaction of transmembrane or secreted ligands with transmembrane receptors. Signalling is also initiated when secreted molecules interact with receptors at a considerable distance from the source of the secreted ligand. Therefore precise intra-cellular trafficking of secreted molecules, ligands and receptors is essential for proper signalling and thus development of the tissue. Signalling by the EGFR ligands is an example illustrating regulation during secretion. A detailed study is required to determine the exact role of Sec $61 \beta$ in secretion of signalling molecules, however what seems to be clear is that the function may not be restricted to the ER. In addition, as demonstrated in the present study, Sec61 $\beta$ appears to provide a fine-tuning in trafficking of specific ligands. This study also illustrates that use of a sensitive in-vivo assay like Gurken signalling in addition to in-vitro systems can provide greater insight into the process of protein translocation.

\section{Conclusion}

The Sec61 channel is the only known protein translocation channel in the ER and is conserved in evolution. In this study we have established the role of the $\beta$ subunit of the channel during plasma membrane traffic of the TGF $\alpha$ like growth factor, Gurken, during Drosophila oogenesis. Gurken signalling is responsible for early patterning in the embryo, absence of Sec61 $\beta$ and the subsequent defects in Gurken trafficking result in dorsalization of the embryo due to reduced Gurken signalling. We also made the surprising observation that although Sec61 $\beta$ is part of the protein transport channel at the ER it affects the transport of Gurken from the Golgi to the plasma membrane. In addition our study provides an excellent in-vivo tool to investigate the process of ER translocation.

\section{Methods}

\section{Drosophila stocks and phenotypic analysis}

The following stocks were used in our experiments:

$y, w$ hs-FLP; FRT42B ovo ${ }^{D(2 R)} / C y O$

$y, w ; F R T 42 B \sec 61 \beta^{p 1} / C y o$

The Golgi Marker $p\{s q h$-EYFP-Golgi $\} 3$ as described in[20]from the Bloomington Stock Center. To generate germline clones, $y$, w;FRT42 sec61 $\beta^{\text {p1 }} / C y o$ females were 
crossed with $y, w$ hs-FLP; FRT42B ovo ${ }^{D(2 R)} / C y O$ males. The progeny were heat-shocked at $37^{\circ} \mathrm{C}$ for 1 hour during the early pupal stage. Ovaries were dissected from females with the genotype $y w$ hs-FLP; FRT42 sec61 $\beta^{\text {P1 }} /$ FRT42 ovo $\mathrm{D}(2 R)$.

\section{In-situ Hybridization}

Ovaries from 1- to 4-day-old females were used for in-situ hybridization with full length, dioxyginin-labeled DNA probe against the Gurken mRNA as described [6].

\section{Immunocytochemistry and microscopy}

Ovaries from 1- to 4-day-old females were dissected in PBS, and kept on ice. Ovaries were fixed in 6:1 Heptane:FIX (Fix: 4\% Formaldehyde in PBS) for 15 minutes. All antibody staining was carried out in PBS, containing $0.5 \%$ BSA, $0.1 \%$ Triton-X-100 and 5\% normal goat serum. The following stains and primary antibodies were used: Texas Red-X/FITC phalloidin, mouse anti-Gurken 1D12 (Hybridoma bank), mouse anti-Sec5 22A2 [24], rat antiYolkless [16], Anti Boca [15], anti-Lava lamp [19]. Secondary antibodies used were FITC, $\mathrm{Cy} 3$ or $\mathrm{Cy} 5$ conjugated (Jackson Laboratories). Confocal data were acquired as single images or image stacks of multi-tracked, separate channels with a Zeiss LSM 510 microscope.

\section{Design of siRNA Constructs in pSupressor}

Sec61 $\beta$ siRNAs were designed and cloned into pSupressor as described in the manufacturer's protocols (Imgenex, San Diego).

\section{The Sec $61 \beta$ oligo used was: TGTTCCAGTATTGGTTATGA}

A scrambled sequence was used as a control: TTAATTTCTGGGAGCTTATG The sequence is identical to both human Sec61 $\beta$ genes, at the 5 ' untranslated region. The oligo contained an intervening sequence that forms the stem-loop structure in the RNA and includes a ScaI site for identification of recombinants. The oligo pair was annealed at 20 $\mu \mathrm{M}$ in annealing buffer $(100 \mathrm{mM}$ potassium acetate, 30 $\mathrm{mM}$ HEPES-KOH (pH 7.4), $2 \mathrm{mM}$ magnesium acetate) at $95^{\circ} \mathrm{C}$ for $4 \mathrm{~min}$, followed by incubation at $70^{\circ} \mathrm{C}$ for 10 min and slow cooling to room temperature. Forty picomoles of annealed oligos were phosphorylated by T4 polynucleotide kinase before they were ligated into pSupressor vector digested by SalI and XbaI.

\section{Transfection and biochemical analysis}

HeLa cells were grown in Dulbecco's Modified Eagle's medium (DMEM) supplemented with 10\% fetal calf serum and Penicillin and Streptomycin (Invitrogen). Transfections in HeLa cells were done using Lipofectamine-2000 following the manufactures protocol (Invitrogen). $3 \mu \mathrm{g}$ of each of the plasmid was used for per $10 \mathrm{~cm}$ cell culture petriplate containing $70-80 \%$ conflu- ent cells. After three days of the first transfection a second transfection was done using the same set of plasmids.

Cells were scrapped off the petriplates and lysis buffer was added [ $1 \%$ Triton, $150 \mathrm{mM} \mathrm{NaCl}, 5 \mathrm{mM}$ EDTA, supplemented with protease inhibitory cocktail (Roche). SDSPAGE and western blotting were done following standard protocols. Endoglycosidase H (Endo-H, New England Biolabs) treatment was done as per manufacturer's instructions. Antibodies used for western blotting were rabbit anti-Drosophila Sec61 $\beta$ (peptide used corresponds to amino acid $2-10$ of the Drosophila sequence, PAPPSSTSV-C), anti-human Sec61 $\beta$ [2], anti-Gurken 1D12 (Hybridoma bank), anti-Myc and anti-HA (Santa Cruz Biotech).

For pulse analysis, cells were depleted of endogenous methionine and cysteine by growing cells in DMEM without methionine and cysteine for two hours before incubation with $100 \mu \mathrm{Ci}$ of ${ }^{35} \mathrm{~S}$ labeled methionine and cysteine per $10 \mathrm{~cm}$ petriplate. The cells were pulsed for 10 minutes, washed with PBS, scraped off the plates and lysis buffer was added. Proteins were immuno-precipitated, and applied to SDS-PAGE after treatment with Endo H treatment.

\section{Authors' contributions}

AK conceived the project and did all the experiments. BD supervised the project. All authors read and approved the final manuscript.

\section{Acknowledgements}

We thank Marek Mlodzik and Bloomington stock center for fly lines and reagents. Matthew Freeman for the Gurken, Star and Rho plasmids. AK thanks Hermann Steller at the Rockefeller University for his generous support. AK also thanks Mala Murthy and Thomas Schwartz at the Harvard Medical School/Children's Hospital for help during the initial stages of the project. AK is currently supported by the Rockefeller University and Howard Hughes Medical Institute.

\section{References}

I. Berg B Van den, Clemons WM Jr, Collinson I, Modis Y, Hartmann E, Harrison SC, Rapoport TA: X-ray structure of a protein-conducting channel. Nature 2004, 427(6969):36-44.

2. Gorlich D, Rapoport TA: Protein translocation into proteoliposomes reconstituted from purified components of the endoplasmic reticulum membrane. Cell 1993, 75(4):615-630.

3. Toikkanen J, Gatti E, Takei K, Saloheimo M, Olkkonen VM, Soderlund $H$, De Camilli P, Keranen S: Yeast protein translocation complex: isolation of two genes SEBI and SEB2 encoding proteins homologous to the Sec6I beta subunit. Yeast 1996, I 2(5):425-438.

4. Panzner S, Dreier L, Hartmann E, Kostka S, Rapoport TA: Posttranslational protein transport in yeast reconstituted with a purified complex of Sec proteins and Kar2p. Cell 1995, 8I(4):56I-570.

5. Valcarcel R, Weber U, Jackson DB, Benes V, Ansorge W, Bohmann $D$, Mlodzik M: Sec6 Ibeta, a subunit of the protein translocation channel, is required during Drosophila development. J Cell Sci 1999, I I 2(Pt 23):4389-4396.

6. Neuman-Silberberg FS, Schupbach T: The Drosophila dorsoventral patterning gene gurken produces a dorsally localized 
RNA and encodes a TGF alpha-like protein. Cell 1993, 75(I): 165-174.

7. Schupbach T, Roth S: Dorsoventral patterning in Drosophila oogenesis. Curr Opin Genet Dev 1994, 4(4):502-507.

8. Freeman M: Proteolysis within the membrane: rhomboids revealed. Nat Rev Mol Cell Biol 2004, 5(3): 188-197.

9. Guichard A, Roark M, Ronshaugen M, Bier E: brother of rhomboid, a rhomboid-related gene expressed during early Drosophila oogenesis, promotes EGF-R/MAPK signaling. Dev Biol 2000, 226(2):255-266.

10. Roth S, Neuman-Silberberg FS, Barcelo G, Schupbach T: cornichon and the EGF receptor signaling process are necessary for both anterior-posterior and dorsal-ventral pattern formation in Drosophila. Cell 1995, 81(6):967-978.

11. Chou TB, Perrimon N: The autosomal FLP-DFS technique for generating germline mosaics in Drosophila melanogaster. Genetics 1996, I44(4): 1673-1679.

12. Nilson LA, Schupbach T: EGF receptor signaling in Drosophila oogenesis. Curr Top Dev Biol 1999, 44:203-243.

13. Saunders C, Cohen RS: The role of oocyte transcription, the 5'UTR, and translation repression and derepression in Drosophila gurken mRNA and protein localization. Mol Cell 1999 , 3(I):43-54

14. Queenan AM, Barcelo G, Van Buskirk C, Schupbach T: The transmembrane region of Gurken is not required for biological activity, but is necessary for transport to the oocyte membrane in Drosophila. Mech Dev 1999, 89(I-2):35-42.

15. Culi J, Mann RS: Boca, an endoplasmic reticulum protein required for wingless signaling and trafficking of LDL receptor family members in Drosophila. Cell 2003, I I 2(3):343-354.

16. Schonbaum CP, Lee S, Mahowald AP: The Drosophila yolkless gene encodes a vitellogenin receptor belonging to the low density lipoprotein receptor superfamily. Proc Natl Acad Sci USA 1995, 92(5): 1485-| 489.

17. Urban S, Lee JR, Freeman M: A family of Rhomboid intramembrane proteases activates all Drosophila membrane-tethered EGF ligands. Embo J 2002, 2 I ( 16):4277-4286.

18. Lee JR, Urban S, Garvey CF, Freeman M: Regulated intracellular ligand transport and proteolysis control EGF signal activation in Drosophila. Cell 200I, 107(2):16I-I7I.

19. Sisson JC, Field C, Ventura R, Royou A, Sullivan W: Lava lamp, a novel peripheral golgi protein, is required for Drosophila melanogaster cellularization. J Cell Biol 2000, I 5 I (4):905-918.

20. Lajeunesse DR, Buckner SM, Lake J, Na C, Pirt A, Fromson K: Three new Drosophila markers of intracellular membranes. Biotechniques 2004, 36(5):784-788.

21. Lipschutz JH, Lingappa VR, Mostov KE: The exocyst affects protein synthesis by acting on the translocation machinery of the endoplasmic reticulum. J Biol Chem 2003, 278(23):20954-20960.

22. Toikkanen JH, Miller KJ, Soderlund H, Jantti J, Keranen S: The beta subunit of the Sec6Ip endoplasmic reticulum translocon interacts with the exocyst complex in Saccharomyces cerevisiae. J Biol Chem 2003, 278(23):20946-20953.

23. Hsu SC, TerBush D, Abraham M, Guo W: The exocyst complex in polarized exocytosis. Int Rev Cytol 2004, 233:243-265.

24. Murthy M, Schwarz TL: The exocyst component Sec5 is required for membrane traffic and polarity in the Drosophila ovary. Development 2004, I 3 I (2):377-388.

25. Helmers J, Schmidt D, Glavy JS, Blobel G, Schwartz T: The beta-subunit of the protein-conducting channel of the endoplasmic reticulum functions as the guanine nucleotide exchange factor for the beta-subunit of the signal recognition particle receptor. J Biol Chem 2003, 278(26):23686-23690.

26. Kalies KU, Rapoport TA, Hartmann E: The beta subunit of the Sec6 I complex facilitates cotranslational protein transport and interacts with the signal peptidase during translocation. J Cell Biol 1998, I4I(4):887-894.

27. Herpers B, Rabouille C: mRNA localization and ER-based protein sorting mechanisms dictate the use of transitional endoplasmic reticulum-golgi units involved in gurken transport in Drosophila oocytes. Mol Biol Cell 2004, I 5( I 2):5306-53 I7.

28. Riechmann V, Ephrussi A: Axis formation during Drosophila oogenesis. Curr Opin Genet Dev 200I, I I (4):374-383.

29. Sans N, Prybylowski K, Petralia RS, Chang K, Wang YX, Racca C, Vicini S, Wenthold RJ: NMDA receptor trafficking through an interaction between PDZ proteins and the exocyst complex. Nat Cell Biol 2003, 5(6):520-530.

30. Wang S, Hsu SC: The molecular mechanisms of the mammalian exocyst complex in exocytosis. Biochem Soc Trans 2006, 34(Pt 5):687-690.

3I. Bokel C, Dass S, Wilsch-Brauninger M, Roth S: Drosophila Cornichon acts as cargo receptor for ER export of the TGFalphalike growth factor Gurken. Development 2006, I33(3):459-470.

32. Tsruya R, Schlesinger A, Reich A, Gabay L, Sapir A, Shilo BZ: Intracellular trafficking by Star regulates cleavage of the Drosophila EGF receptor ligand Spitz. Genes Dev 2002, I 6(2):222-234.
Publish with Biomed Central and every scientist can read your work free of charge

"BioMed Central will be the most significant development for disseminating the results of biomedical research in our lifetime. "

Sir Paul Nurse, Cancer Research UK

Your research papers will be:

- available free of charge to the entire biomedical community

- peer reviewed and published immediately upon acceptance

- cited in PubMed and archived on PubMed Central

- yours - you keep the copyright
BioMedcentral 